\title{
Metodologia para aplicação de técnicas de Gamificação e Planta Virtual no curso de Automação
}

\author{
Marcos Vinícius de Amorim ${ }^{1}$, Eduardo Lorenzetti Pellini ${ }^{1}$ \\ ${ }^{1}$ Departamento de Engenharia de Energia e Automação Elétricas \\ Escola Politécnica da Universidade de São Paulo (USP) - São Paulo, SP - Brasil \\ marcos_amorim@usp.br, elpellini@usp.br
}

\begin{abstract}
Automation is present in several industrial processes, controlling variables and executing sequential tasks, in order to obtain an optimized and standardized process. So, for students in this area, the practical part is very important, but there is a need for a high financial value for institutions to maintain teaching laboratories. Therefore, in this work, a Virtual Plant of a Varnishes Factory is presented, where students can apply the techniques studied in theory. In addition, to improve students' motivation and engagement, Gamification techniques were used in experimental scripts in the platform Moodle and in the Virtual Plant developed in this work.
\end{abstract}

Resumo. A automação está presente em diversos processos industriais, controlando variáveis e executando tarefas sequenciais, com a finalidade de obter um processo otimizado e padronizado. Portanto, para os alunos dessa área, a parte prática é muito importante, porém necessita-se um alto valor financeiro para as instituições manterem os laboratórios didáticos. Logo, é apresentado nesse trabalho uma Planta Virtual de uma Fábrica de Vernizes, onde os alunos podem aplicar as técnicas estudadas na teoria. Além disso, para melhorar a motivação e o engajamento dos alunos, utilizaram-se técnicas de Gamificação nos roteiros experimentais, na plataforma Moodle e na Planta Virtual elaborada nesse trabalho.

\section{Introdução}

A automação está presente em grande parte dos processos industriais, controlando variáveis do sistema ou executando tarefas sequenciais, tornando o processo automatizado [Araujo and Carreiro 2017]. Inicialmente, a automação tinha como objetivo apenas monitorar e controlar localmente os principais parâmetros de um sistema, porém atualmente, a automação está presente em ambientes insalubres para a presença humana e onde se busca otimização e padronização de um processo obtendo redução de custos, eficiência na produção, aumento de qualidade dos produtos e integração dos sistemas [Tagliari et al. 2010]. Ela surgiu durante a Terceira Revolução Industrial, logo após a Segunda Guerra Mundial, onde foram desenvolvidas novas tecnologias com o propósito de diminuir a participação humana nos processos industriais. Nessa época surgiram os componentes eletrônicos nas máquinas, Controladores Lógicos Programáveis (CLP), robôs e a automação começou a trabalhar em conjunto com as Tecnologias da Informação (TI) integrando processos, máquinas e pessoas. Atualmente, estamos vivenciando a Quarta Revolução Industrial, também conhecida como Indústria 4.0, onde as máquinas estão integradas junto à internet [Dos Santos et al. 2018] e com o tempo, os processos de fabricação 
estão evoluindo de uma única célula automatizada para sistemas totalmente automatizados e integrados, melhorando a flexibilidade, velocidade, produtividade e qualidade dos sistemas produtivos [Jr et al. 2017].

Geralmente, os laboratórios de ensino de automação utilizam uma grande variedade e quantidade de equipamentos, como CLPs, módulos didáticos, plantas-pilotos e diversos sensores e atuadores, a fim de permitir o desenvolvimento de atividades práticas de projeto, implementação e teste de diferentes técnicas vistas na teoria [Persechini and Mendes 2014]. Porém, umas das maiores dificuldades dos estudantes de engenharia é justamente o acesso às atividades práticas. Isso ocorre devido ao elevado número de estudantes que necessitam utilizar os equipamentos disponíveis, sendo que para aumentar o número de equipamentos é necessário um elevado investimento das instituições, não somente na aquisição, mas também em infraestrutura e manutenção periódica.

Para minimizar esses problemas, uma das soluções é o desenvolvimento de Plantas Virtuais que mesmo não oferecendo todos os recursos das plantas físicas, se bem estruturadas, podem ter ótimos resultados [Araujo and Carreiro 2017]. O modelo de uma planta é uma representação total ou parcial de um sistema físico, que é utilizado para simular o comportamento da planta em seu ponto de operação. Além disso, pode haver vários modelos para representar um mesmo sistema físico, com diferentes níveis de fidelidade, dependendo dos fenômenos de interesse e para qual problema foi destinado [Canadas et al. 2018].

Outro ponto a ser mencionado com relação à educação é a falta de engajamento e o desinteresse dos alunos durante as aulas. Esse desafio cresce conforme as tecnologias evoluem e os métodos de ensino continuam inalterados. Por isso, as instituições de ensino e seu corpo técnico buscam soluções para reter a atenção dos alunos e melhorar a eficiência do processo de aprendizagem [Boaventura and Oliveira 2018].

Realizar atividades baseadas em jogos, como os presentes em esportes, brincadeiras e jogos eletrônicos, conseguem manter a atenção das pessoas de forma que as mesmas passam horas concentradas numa atividade de alto desempenho [Fraga et al. 2016]. Neste cenário surgiu a Gamificação, do inglês Gamification, que é o uso de técnicas de jogos aplicadas em ambientes sérios. Definida como a utilização de elementos de jogos fora do contexto de jogo, a Gamificação pode ser utilizada para aprimorar a motivação e o engajamento no processo de aprendizagem [Toda et al. 2017].

Baseado nisso, este trabalho tem como objetivo projetar uma Planta Virtual que utilize tecnologias da Indústria 4.0 para ser utilizada no ensino na área de Automação e Controle, onde os alunos poderão verificar experimentalmente os conceitos técnicos vistos nas aulas teóricas. Além disso, é proposto um ambiente de aprendizagem utilizando elementos de Gamificação com o intuito de tornar as aulas mais dinâmicas e motivadoras aos alunos.

\section{Metodologia}

Este trabalho consiste em elaborar uma Planta Virtual que faz parte de uma Fábrica de Vernizes onde cada célula de produção estará numa bancada, operando de forma individual ou em conjunto. Como base para esse projeto, foram utilizados os seguintes kits didáticos disponíveis no laboratório: 
- Kit do CLP CompactLogix;

- Kit da Interface Humano-Máquina (IHM) PanelView Plus 1000;

- Giga de teste de um reator químico, conforme Figura 1.

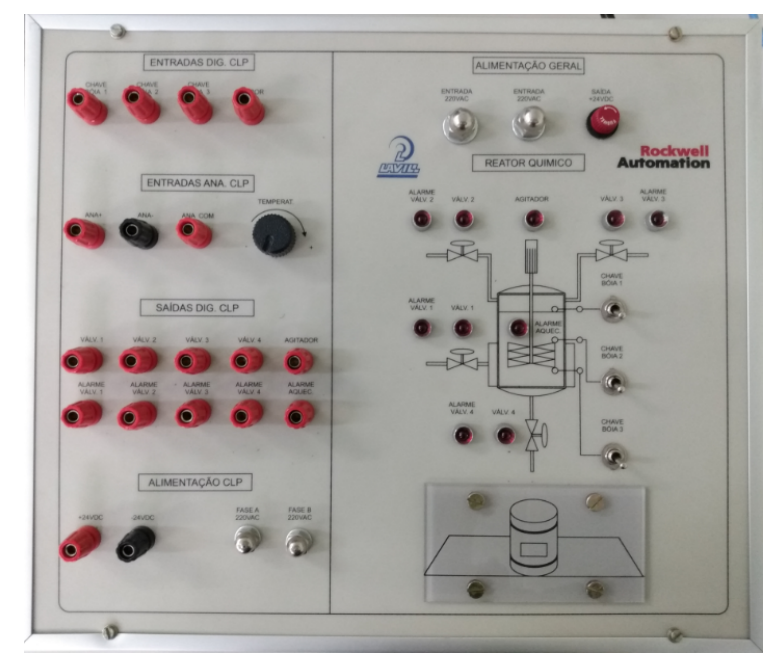

Figura 1. Giga de teste do reator químico disponível no laboratório

Observa-se nessa figura que o funcionamento do reator depende de operações manuais através de potenciômetros e chaves elétricas. Logo, o intuito deste trabalho é elaborar uma Planta Virtual que execute estas ações automaticamente, substituindo a Giga de teste, possibilitando a dedicação dos alunos no comissionamento do CLP.

Para criar uma Planta Virtual é necessário elaborar um modelo matemático de uma planta real, porém é praticamente impossível descrever todos os aspectos dela devido a sua complexidade. Por consequência, é preciso selecionar quais são as características necessárias e suficientes para descrever um determinado comportamento desta planta e traduzir para um conjunto de equações matemáticas. Em seguida, modelar e validar essa Planta no software MatLab. Finalmente, para realizar o controle em tempo real dessa planta, transcrever para o LabVIEW que estará conectado a um CompactRIO da National Instruments.

O laboratório possui seis bancadas e cada uma contém: um CLP, uma IHM, um CompactRIO e dois computadores (um para o desenvolvimento e outro para executar a Planta Virtual). Esses equipamentos foram utilizados para formar um simulador do tipo HiL (Hardware in the Loop), ou seja, um simulador com uma Planta Virtual controlada por um CLP real, possuindo os sensores e atuadores apresentados na Tabela 1, vinculados aos cartões de entrada e saída do CompactRIO. Além disso, o CLP está conectado com a IHM, permitindo um maior controle do aluno sobre a planta.

Como um dos objetivos da Indústria 4.0 é a conectividade, as bancadas podem trabalhar em conjunto, existindo um sétimo computador onde é executado um Sistema Supervisório, responsável em gerenciar as plantas das seis bancadas.

Além disso, para tornarem as aulas mais dinâmicas foram utilizados elementos de Gamificação nas aulas e no Ambiente Virtual de Aprendizagem (AVA) Moodle. É proposto um projeto de automação desta Fábrica de Vernizes, dividido em várias etapas, onde o nível de dificuldade é incrementado até que o projeto final seja atingido. Cada etapa tem 
IX Congresso Brasileiro de Informática na Educação (CBIE 2020)

Anais do XXXI Simpósio Brasileiro de Informática na Educação (SBIE 2020)

Tabela 1. Lista de sensores e atuadores da célula de produção

\begin{tabular}{|c|c|}
\hline ELEMENTO & DESCRIÇÃO \\
\hline \multicolumn{2}{|r|}{ Sensores digitais } \\
\hline Boia 1 & Sensor do tipo boia indicando nível do tanque de $1 \%$ \\
\hline Boia 2 & Sensor do tipo boia indicando nível do tanque de $25 \%$ \\
\hline Boia 3 & Sensor do tipo boia indicando nível do tanque de $50 \%$ \\
\hline Boia 4 & Sensor do tipo boia indicando nível do tanque de $75 \%$ \\
\hline Boia 5 & Sensor do tipo boia indicando nível do tanque de $99 \%$ \\
\hline Tambor de Armazenamento & Tambor utilizado para retirada do verniz \\
\hline \multicolumn{2}{|r|}{ Atuadores digitais } \\
\hline Válvula 1 & Válvula de controle da entrada de resina no tanque \\
\hline Válvula 2 & Válvula de controle da entrada do aditivo 1 \\
\hline Válvula 3 & Válvula de controle da entrada do aditivo 2 \\
\hline Válvula 4 & Válvula de controle da saída do tanque \\
\hline Agitador & Hélice acoplada a um motor para realizar a mistura \\
\hline \multicolumn{2}{|r|}{ Sensores analógicos } \\
\hline Sensor de Temperatura & Sensor para leitura da temperatura do tanque \\
\hline Sensor de Nível & Sensor para leitura do nível da mistura no tanque \\
\hline \multicolumn{2}{|r|}{ Atuador analógico } \\
\hline Válvula Proporcional & Válvula para contr \\
\hline
\end{tabular}

um sistema de pontuação com relação às atividades realizadas e a soma destes pontos será utilizada para auxiliar o conceito final dos alunos. Também foram utilizadas algumas ferramentas disponíveis no Moodle em conjunto com as motivações da framework Octalysis proposta por Yu-Kai-Chou [Chou 2015].

\section{Resultados e Discussões}

Foram utilizados conceitos de Termodinâmica, Mecânica dos Fluidos e Modelagem de Sistemas Dinâmicos para definir as características desejadas para este modelo. Por se tratar de um tanque de mistura de líquidos onde ocorre troca de calor, ele pôde ser tratado como um Volume de Controle de um Sistema Termohidráulico. Baseado nisso, inicialmente, estudou-se somente o comportamento hidráulico do sistema, adotando as seguintes premissas [Felício 2010]:

- O fluido é incompressível, logo, tem massa específica constante;

- A temperatura no fluido é uniforme;

- As resistências fluídicas são localizadas, constantes e lineares;

- O tanque tem a seção de área uniforme e constante;

- A pressão atmosférica é constante;

- Os efeitos de inércia do fluido são desprezíveis;

- As variações das grandezas são relativamente pequenas, a fim de manter o comportamento linear do sistema.

Em seguida, utilizou-se a Lei de Conservação da Massa como base do modelo, obtendo a primeira equação de movimento do sistema: 


$$
V(s)=\frac{F_{i n}-\frac{\mu g h}{R_{f}}}{s}
$$

Onde: $V=$ Volume do tanque em $m^{3} ; F=$ Vazão do líquido em $\mathrm{m}^{3} / \mathrm{s} ; \mu=$ Massa específica do líquido em $\mathrm{kg} / \mathrm{m}^{3} ; g=$ Gravidade em $\mathrm{m} / \mathrm{s}^{2} ; h=$ Altura do tanque em $\mathrm{m}$; $R_{f}=$ Resistência fluídica em $\mathrm{kg} / \mathrm{m}^{4} \mathrm{~s}$.

Em seguida, foi estudado o efeito termodinâmico no sistema, adotando as seguintes premissas [Garcia 2009]:

- Massa específica dos fluidos sendo aquecidos é sempre constante;

- Capacitância térmica da serpentina de aquecimento desprezível;

- O vapor de aquecimento entra e permanece saturado e sua pressão é constante;

- O purgador de vapor é considerado ideal e elimina todo o condensado que se forma;

- O calor específico dos fluidos de processo é constante;

- As resistências e capacitâncias térmicas são constantes e lineares [Felício 2010];

- Os materiais sempre ficam no mesmo estado de fase.

Baseado nessas premissas, aplicou-se a Lei de Conservação de Energia e foi considerado outros elementos, além do fluido do Volume de Controle, que podem afetar o balanço de energia que são: o fluido do vapor saturado, a perda natural de temperatura para o ambiente e a perda de temperatura causada pelo agitador. Com isso, foi obtida a segunda equação de movimento do modelo:

$$
\begin{gathered}
T_{\text {tanque }}(s)=T_{\text {amb }}+\frac{\mu F_{\text {in }} c\left(T_{\text {in }}-T_{\text {tanque }}\right)-\mu F_{\text {out }} c\left(T_{\text {tanque }}-T_{\text {amb }}\right)+\mu F_{\text {vap }} c\left(T_{\text {vap }}-T_{\text {tanque }}\right)}{m c s} \\
\frac{-U A_{\text {nat }}\left(T_{\text {tanque }}-T_{\text {amb }}\right)-U A_{\text {agit }}\left(T_{\text {tanque }}-T_{\text {amb }}\right)}{m c s}
\end{gathered}
$$

Onde: $T=$ Temperatura em ${ }^{\circ} C ; c=$ Calor específico do líquido em $\mathrm{J} / \mathrm{kg}^{\circ} \mathrm{C}$; $m=$ Massa do líquido em $\mathrm{kg}$; $U A=$ Coeficiente global de transferência térmica em $W /{ }^{\circ} \mathrm{Cm}^{2}$

Com base nas equações de movimento, descritas em 1 e 2, foi feito um programa no MatLab utilizando a ferramenta Simulink, para testar e validar o modelo, onde foram adotados os seguintes parâmetros:

- Resistência fluídica: $750000 \mathrm{~kg} / \mathrm{m}^{4} \mathrm{~s}$;

- Gravidade: $10 \mathrm{~m} / \mathrm{s}^{2}$;

- Massa específica: $10 \mathrm{~kg} / \mathrm{m}^{3}$;

- Calor específico: $1 \mathrm{~J} / \mathrm{kg}^{\circ} \mathrm{C}$;

- Área: $1 m^{2}$;

- Vazão de entrada: $400 \mathrm{l} / \mathrm{min}=0,4 \mathrm{~m}^{3} / \mathrm{min}$;

- Massa do tanque: $100 \mathrm{~kg}$;

- Volume máximo: $1000 l=1 \mathrm{~m}^{3}$;

- Vazão do vapor máxima: $2000 l / \min =2 \mathrm{~m}^{3} / \min$; 
IX Congresso Brasileiro de Informática na Educação (CBIE 2020)

Anais do XXXI Simpósio Brasileiro de Informática na Educação (SBIE 2020)

- Perda de calor natural do tanque: $4 J$;

- Perda de calor do tanque com o agitador ligado: $15 \mathrm{~J}$.

Para validar essa simulação, foi realizado o seguinte teste:

1. Condição inicial com as válvulas fechadas e temperatura ambiente à $20^{\circ} C$;

2. Abriu-se uma válvula de entrada para encher o tanque com o fluido à $40^{\circ} \mathrm{C}$, até chegar em $500 l$ aproximadamente;

3. Abriu-se totalmente a válvula proporcional, aquecendo o tanque com o vapor à $150^{\circ} \mathrm{C}$ e aguardou-se a estabilização;

4. Ligou-se o agitador até a temperatura estabilizar novamente;

5. Fechou-se a válvula proporcional e após um tempo, desligou-se o agitador;

6. Abriu-se a válvula de saída do tanque;

7. Aguardou-se o tanque esvaziar e a temperatura voltar à temperatura ambiente.

Utilizando um tempo de amostragem de $0,1 \mathrm{~ms}$, foi obtido o resultado da Figura 2, onde o modelo foi validado, pois se comportou conforme o desejado.

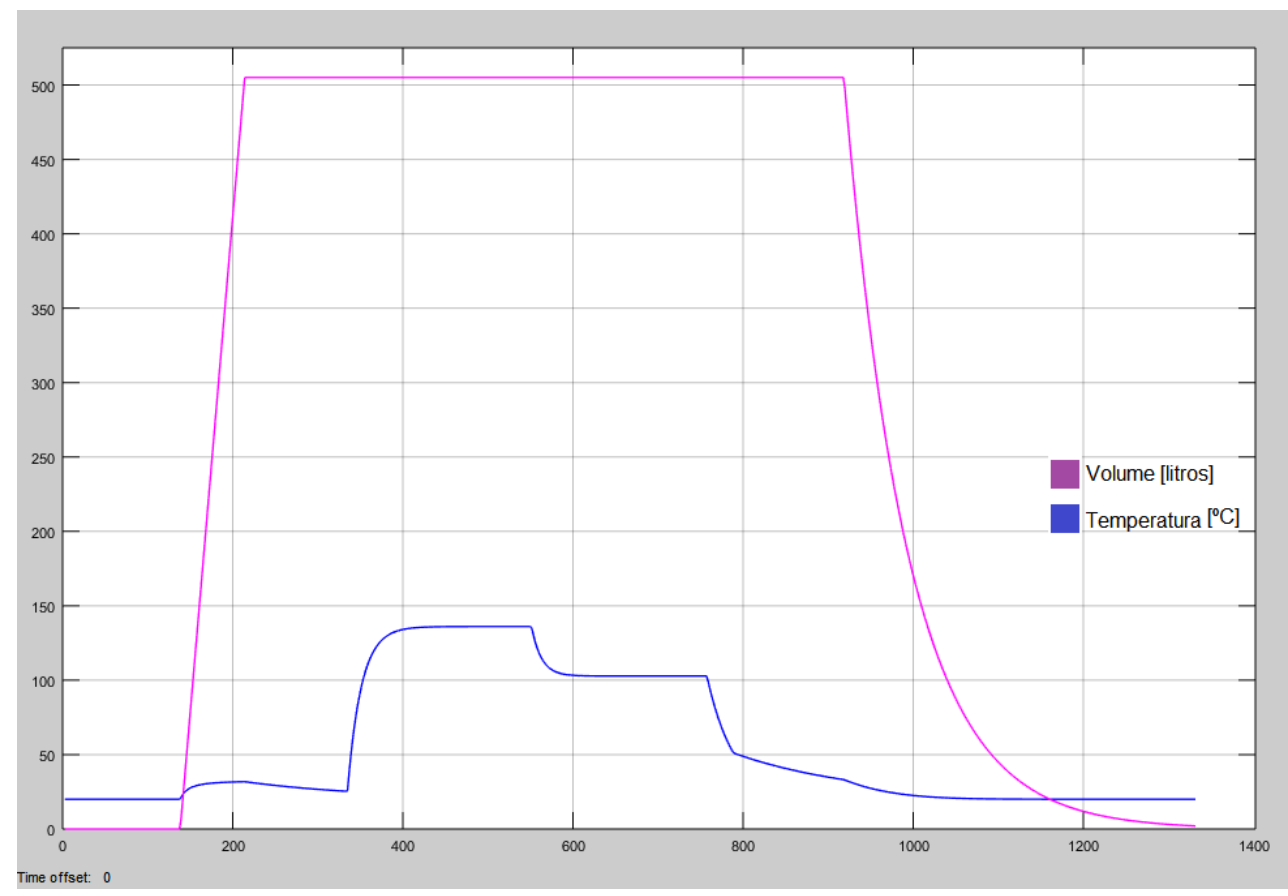

Figura 2. Resultado da simulação feita no MatLab

Para criar a Planta Virtual foi utilizado o software LabVIEW, versão 2014, e instalado os módulos adicionais: Real-Time Module e Control Design and Simulation Module. Em seguida, foi feito a transcrição do diagrama feito no Simulink para o ambiente LabVIEW no Block Diagram. Destaca-se que para rodar um bloco de integração na resposta em frequência, Integrator, foi necessário executar todo o programa dentro de um Control and Simulation Loop.

O modelo da planta fica disponível no Block Diagram, porém os alunos não têm acesso, sendo disponibilizado para eles apenas o Front Panel que contém a animação da Planta Virtual. Para criar uma interface intuitiva, foi desenhado o fundo de tela e acrescentado os elementos do LabVIEW, obtendo o Front Panel mostrado na Figura 3. 
IX Congresso Brasileiro de Informática na Educação (CBIE 2020)

Anais do XXXI Simpósio Brasileiro de Informática na Educação (SBIE 2020)

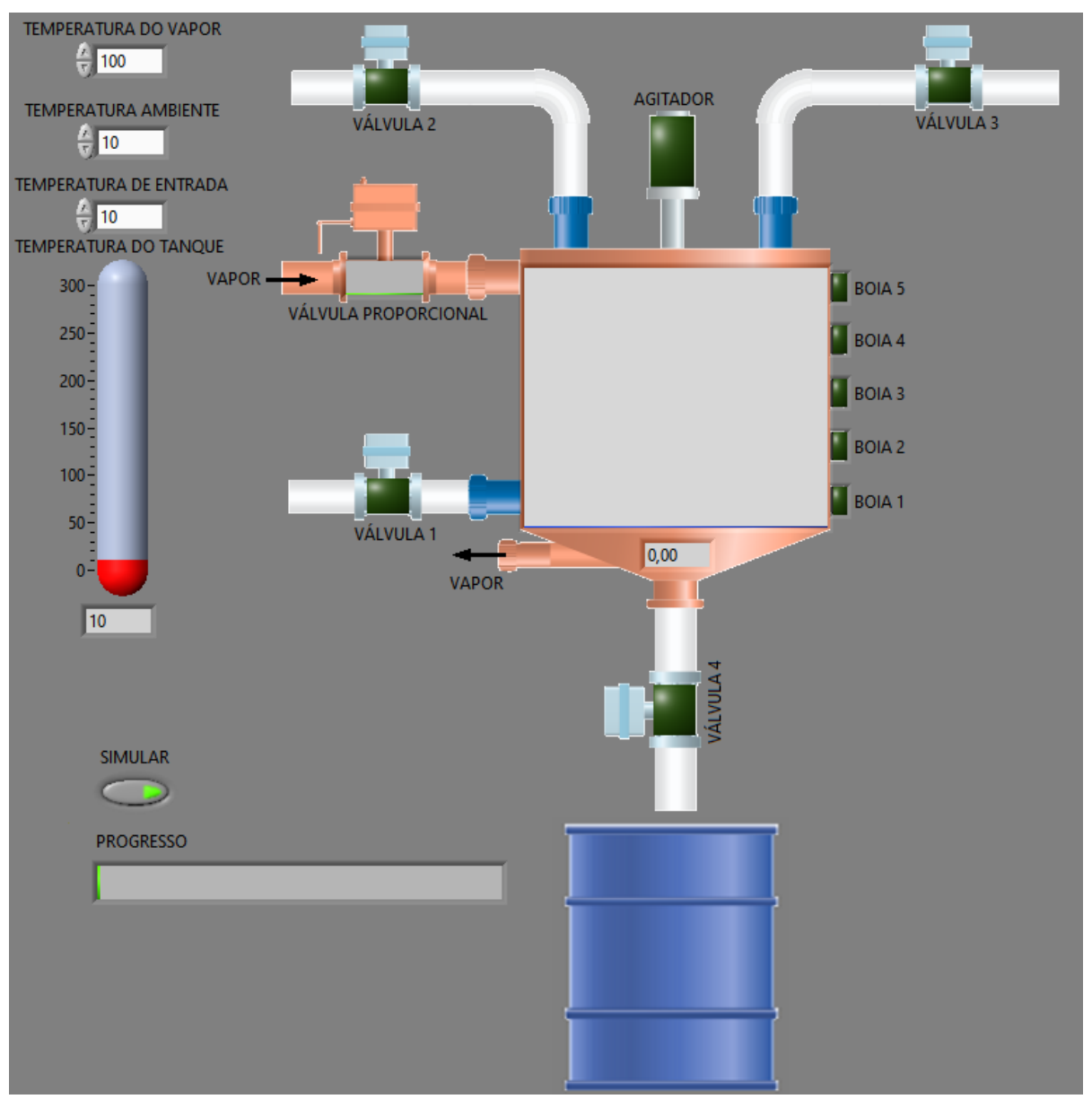

Figura 3. Front Panel da Planta Virtual

Observa-se nessa figura, os elementos de entrada e saída da planta, conforme a Tabela 2:

Tabela 2. Lista dos elementos da Planta Virtual

\begin{tabular}{|c|c|}
\hline ELEMENTO & DESCRIÇÃO \\
\hline Boias & Indicador booleano. Verde escuro (desacionado) ou claro (acionado) \\
\hline Tambor & Indicação de presença. Visível ou não \\
\hline Válvulas & Indicador booleano. Verde escuro (fechada) ou claro (aberta) \\
\hline Agitador & Indicador booleano. Verde escuro (desligado) ou claro (ligado) \\
\hline Temp. Tanque & Bar Graph que indica a temperatura do tanque \\
\hline Válvula Prop. & Bar Graph que indica o nível de abertura da válvula \\
\hline Tanque & Bar Graph que indica o nível do tanque \\
\hline Temp. Entrada & Controle numérico com o valor da temperatura dos fluidos na entrada \\
\hline Temp. Ambiente & Controle numérico com o valor da temperatura ambiente \\
\hline Temp. Vapor & Controle numérico com o valor da temperatura do vapor na entrada \\
\hline Simular & Botão para iniciar e parar a simulação \\
\hline Progresso & Bar Graph que indica o quanto que o processo foi realizado \\
\hline
\end{tabular}

Para validar essa planta, foi realizado o mesmo teste feito no MatLab e verificou- 
IX Congresso Brasileiro de Informática na Educação (CBIE 2020)

Anais do XXXI Simpósio Brasileiro de Informática na Educação (SBIE 2020)

se que ela se comportou conforme o desejado. Com isso, suas entradas e saídas foram vinculadas com o CompactRIO, para ser integrada ao mundo real, obtendo uma simulação Hardware in the Loop.

O controle dessa planta pode ser feito por diversos tipos de controladores ou acionamentos, porém neste trabalho, o controlador utilizado foi o Kit de CLP disponível e sugerido três roteiros experimentais:

- Bancada autônoma: onde o controle da planta é realizado pelo kit didático do CLP, programado pelo software RSLogix 5000. Através desse kit, o ciclo de funcionamento da planta e o controle de temperatura são executados. Os acionamentos e monitoramentos são feitos, respectivamente, através das chaves e lâmpadas presentes no kit;

- Bancada com IHM local: contém o kit didático do CLP e da IHM, onde é realizado o monitoramento da planta localmente. Através dessa IHM são apresentados os valores de temperatura, volume e contadores, além da seleção do produto a ser produzido na planta, alterando os dados do processo;

- Bancada com IHM remota: tem o objetivo de aumentar a conectividade das bancadas através do monitoramento e gerenciamento das plantas de forma remota, utilizando um Sistema Supervisório. Através desse sistema, todas as bancadas são monitoradas simultaneamente via rede, possibilitando selecionar o tipo de produto em cada planta e verificar o seu estado de funcionamento.

Na segunda etapa deste trabalho são mostradas as ferramentas utilizadas para criar um ambiente de aprendizagem gamificado, tomando como base as bancadas sugeridas e exemplos das referências bibliográficas [Ferreira et al. 2018]. Para isso, foram utilizadas algumas táticas da framework Octalysis nos roteiros experimentais, no ambiente Moodle e na Planta Virtual elaborada neste trabalho, apresentadas a seguir:

- Narrative: Apresentar aos alunos as razões da disciplina, a sua importância e procurar envolvê-los, conforme as aulas forem progredindo através do Moodle, durante as aulas teóricas e nos roteiros experimentais;

- Status Points: Dividir as atividades em missões e atribuir pontuação. No ambiente Moodle pode-se atribuir pesos para cada atividade realizada e gerar um Relatório de Conclusão das Atividades. No laboratório pode-se utilizar um Check List para verificar se os alunos realizaram o experimento, conforme o roteiro da aula;

- Progress Bar: Utilizar no Moodle um bloco chamado Progresso de Conclusão, onde o aluno tem um feedback da sua evolução da matéria com relação a todas as atividades executadas. Além disso, na Planta Virtual existe uma Barra de Progresso que é incrementada quando o programa do aluno atinge um determinado objetivo;

- Blank Fills: Criar algumas lacunas nos programas dos roteiros experimentais, para que os alunos possam completá-las, utilizando os conceitos vistos, desenvolvendo o raciocínio lógico e a criatividade deles;

- Real-Time Control: Fornecer aos alunos um feedback em tempo real das suas ações, através da Planta Virtual;

- Water Cooler: Utilizar fóruns para que os alunos possam se integrar e compartilhar ideias. Para isso, utilizar no Moodle um fórum do tipo Aviso para que o professor possa enviar mensagens importantes com relação a disciplina e utilizar 
um fórum do tipo Geral, onde o professor e os alunos possam criar perguntas e respostas referentes a matéria;

- Envolved UI: Tem como objetivo criar um ambiente de interface evolutiva, ou seja, as informações são apresentadas aos alunos conforme eles vão progredindo com as atividades. Para isso, é necessário utilizar no Moodle as ferramentas de Acesso Condicionado e Restrições e no laboratório utilizar a Planta Virtual que altera suas variáveis, conforme os comandos do usuário;

- MiniQuests: Criar atividades extras durante a disciplina, como questionários;

- CountDown Timers: São atividades que os alunos precisam fazer em um período determinado, que são desabilitadas depois desse período. Para isso, é fundamental usar a ferramenta de Restrição de Acesso por Tempo;

- Collection Sets: Por fim, apresentar alguns Emblemas que os alunos podem obter, conforme a conclusão das atividades no Moodle.

Entre essas táticas, destaca-se a elaboração de uma Barra de Progresso na Planta Virtual. No Block Diagram foi acrescentada uma Máquina de Estados, onde cada estado somente é alterado após a Planta Virtual alcançar uma condição pré-determinada. Para isso, foi fundamental ler todas as variáveis de entrada e saída da planta, a fim de conhecer seu estado atual e em seguida, utilizou-se uma Case Structures, onde cada Case é um Estado, e a mudança somente ocorre quando todas as condições das variáveis são satisfeitas.

\section{Conclusão}

Este artigo apresentou duas técnicas para auxiliar no ensino na área de automação. A primeira foi a elaboração de uma Planta Virtual que simula uma célula de produção de uma Fábrica de Vernizes que foi modelada e validada no MatLab, usando equações de Termodinâmica e Mecânica dos Fluidos, e em seguida, foi implementada no LabVIEW e conectada a um CompactRIO, permitindo a planta ser controlada por um CLP real. Além disso, foram sugeridos três roteiros experimentais utilizando em conjunto com o CLP, uma IHM local e um Sistema Supervisório para monitorar todas as bancadas.

A segunda técnica foi a utilização da Gamificação, que consiste em utilizar elementos de jogos em ambientes que não sejam de jogos, onde foram utilizadas dez táticas da framework Octalysis proposta por Yu-Kai-Chou. Observou-se que é possível aplicar essas táticas não somente nos roteiros experimentais, mas também no ambiente Moodle, utilizando recursos já disponíveis nessa plataforma, e na própria Planta Virtual elaborada nesse trabalho.

Nessa época de pandemia, gerada pelo Covid-19, essas duas técnicas mostram-se como ferramentas auxiliares para as aulas remotas, principalmente nas disciplinas com atividades nos laboratórios, como as que ocorrem no curso de Automação.

Este artigo faz parte de uma pesquisa de Mestrado que está em andamento e para a sua conclusão, serão utilizadas essas técnicas na disciplina PEA3509 - Laboratório de Automação e Proteção de Sistemas Elétricos e Industriais. No final da disciplina será aplicada uma enquete para obter um feedback dos alunos com relação à utilização desta metodologia proposta. 
IX Congresso Brasileiro de Informática na Educação (CBIE 2020)

Anais do XXXI Simpósio Brasileiro de Informática na Educação (SBIE 2020)

\section{Referências}

Araujo, F. A. T. and Carreiro, F. B. (2017). Laboratório semi-virtual para aprendizagem interativa de automação e controle de processos industriais. Congresso Brasileiro de Educação em Engenharia, XLV, Joinville, Anais.

Boaventura, E. F. and Oliveira, R. d. C. S. (2018). Gamificação: Uma análise de sua aplicação como ferramenta de engajamento, aprendizagem e interação em ambientes virtuais. Revista Brasileira de Educação e Cultura-RBEC-ISSN 2237-3098, (17):104-128.

Canadas, N., Machado, J., Soares, F., Barros, C., and Varela, L. (2018). Simulation of cyber physical systems behaviour using timed plant models. Mechatronics, 54:175185.

Chou, Y. K. (2015). Actionable gamification: Beyond points, badges, and leaderboards. Packt Publishing Ltd.

Dos Santos, M., Martins Manhães, A., and Lima, A. R. (2018). Indústria 4.0: desafios e oportunidades para o brasil. Sergipe. SIMPROD.

Felício, L. C. (2010). Modelagem da dinâmica de sistemas e estudo da resposta. RiMa, São Carlos.

Ferreira, M., Morgado, L., and Miranda, G. (2018). Análise das funcionalidades de gamificação nos ambientes de aprendizagem classcraft e moodle à luz da framework octalysis. Lisboa.

Fraga, R. P., Perez, G., and Damin, F. (2016). Situating gamification in relation to other game based concepts: A systematic review. In Proceeding of the 14th Contecsi International Conference on Information Systems and Technology Management, pages 1523-1544, São Paulo. TECSI.

Garcia, C. (2009). Modelagem e Simulação de Processos Industriais e de Sistema Eletromecânicos. Editora da Universidade de São Paulo, São Paulo.

Jr, D., Albertin, M., Elienesio, M., A. S., A., Pontes, H., and Dos Santos Aires, A. (2017). Principais inovações tecnológicas da indústria 4.0 e suas aplicações e implicações na manufatura. Bauru. SIMPEP.

Persechini, M. A. M. and Mendes, L. T. S. (2014). Plataforma integrada para ensino de controle e automação. Congresso Brasileiro de Automática, XX, Belo Horizonte, Anais, pages 1411-1418.

Tagliari, I. B., Junior, G. B., Rosa, L. C., Marques, M. A., de Lemos, M. A., and Sorocaba, A.-C. (2010). Automação em processos produtivos baseada em instrumentação virtual. In 9th IEEE/IAS International Conference on Industry Applications, pages 1-6, Sorocaba. INDUSCON.

Toda, A. M., da Silva, A. P., and Isotani, S. (2017). Desafios para o planejamento e implantação da gamificação no contexto educacional. RENOTE, 15(2). 